\title{
Anisotropy in the Terahertz Band
}

\author{
E. Castro-Camus
}

Received: 8 July 2013/ Accepted: 10 July 2013/

Published online: 3 September 2013

(C) Springer Science+Business Media New York 2013

Day after day, anisotropic properties of materials are finding new applications. Many consumer technologies based on them are currently available, particularly at optical, near-infrared and ultraviolet frequencies. Liquid crystals are the base of an enormous quantity of display devices present in our everyday life ranging from small wrist watches to large television screens, the circular dichroism exhibited by a number of proteins and nucleic acids is providing new information about their molecular structure and biological function, Pockels cells are being used as high speed modulators in optical fiber telecommunications among many other applications. The availability of new technologies to access the terahertz band opened the possibility to study anisotropic properties of materials in the far-infrared which have attracted considerable attention over the last decade owing to their enormous scientific and technological potential.

This special issue contains five invited review articles that cover the most important aspects of the study of anisotropic properties of materials in the terahertz band. The articles included in the issue, enumerated below, represent an up-to-date overview of advances in this field.

1. Recent advances in birefringence studies at THz frequencies by $\mathrm{K}$. Wiesauer and C. Jördens from Research Center for Non-destructive Testing (Austria) and the Technische Universität Braunschweig (Germany) provides an overview that includes instrumental advances, analysis techniques and experiments for the extraction of birefringence of materials from time-domain spectroscopic data.

2. Developments in $\mathrm{THz}$ range ellipsometry by M. Neshat and P. Armitage from the University of Tehran (Iran) and Johns Hopkins University (USA) presents

E. Castro-Camus ( $\triangle$ )

Centro de Investigaciones en Óptica A.C., Loma del Bosque 115, Lomas del Campestre, León,

Guanajuato 37150, México

e-mail: enrique@cio.mx 
an overview on the implementation of terahertz time-domain ellipsometry and recent experiments for the determination of complex refractive indices of a variety of materials using this technique.

3. Towards Dynamic, Tunable, and Nonlinear Metamaterials via Near Field Interactions: A Review G. R. Keiser et al. from R. Averitt's group at Boston University (USA) discusses recent advances and applications of anisotropic metamaterials for various applications such as electron spin resonance.

4. Review of anisotropic terahertz material response by T. Arikawa et al. from J. Kono's group at Rice University (USA) presents a review that includes a phenomenological discussion of the origin of anisotropy, followed by the presentation of a number of experiments in which aligned carbon nanotubes and other materials are studied.

5. Polarization-sensitive THz-TDS and its application to anisotropy sensing by T. Nagashima, M. Tani, and M. Hangyo from the University of Osaka and University of Fukui (Japan) provides an informative review on state-of-the-art instrumental developments that are currently being used for polarimetry and ellipsometry in the terahertz band. 